\title{
LAS HABILIDADES SOCIALES EN NIÑOS PREESCOLARES EN CONTEXTOS DE POBREZA
}

\section{SOCIAL ABILITIES IN PRE-SCHOOL CHILDREN IN POVERTY ENVIRONMENTS}

\author{
Ana Betina Lacunza \\ Universidad Nacional de Tucumán, Argentina \\ Norma Contini de González \\ Universidad Nacional de Tucumán, Argentina
}

\begin{abstract}
Resumen: Las investigaciones han encontrado sólidas relaciones entre la competencia social en la infancia y el funcionamiento social posterior. El estudio de las habilidades sociales en la infancia está centrado en prevenir dificultades de adaptación y posibilitar el desarrollo de recursos personales en diferentes contextos.

El objetivo de este trabajo fue describir una serie de habilidades sociales en 318 niños de 3 a 5 años de S.M. de Tucumán (Argentina) en situación de pobreza.

Se aplicó una Escala de Habilidades Sociales a los padres y una encuesta sociodemográfica, considerándose el nivel socioeconómico.

Los resultados no identificaron diferencias estadísticas significativas según los niveles de pobreza y el sexo de los niños. Posteriormente se analizó la cualidad de las habilidades sociales descriptas. Los resultados permiten afirmar que a pesar del contexto de pobreza, los niños participantes mostraron una serie de habilidades sociales positivas, por lo que se las considera un recurso de salud.
\end{abstract}

Palabras clave: habilidades sociales, niño preescolar, pobreza.

\begin{abstract}
Research has shown a close relationship between social competence in childhood and the subsequent social performance. The study of social abilities in childhood focuses on preventing difficulties with adaption and facilitating the development of personal resources in different contexts.

The aim of this work was to describe a series of social abilities in 318 children between 3 and 5 years old from San Miguel de Tucumán, Argentina who live under poverty conditions.

Taking into consideration the socioeconomic level, a Scale on Social Abilities and a sociodemographic survey were administered to parents.

The results showed no statistically significant differences in relation to poverty levels and the children's sex. The quality of the social abilities described was subsequently analyzed. The results show that, despite the context of poverty, the children involved showed positive social abilities; therefore, these abilities are considered as a health resource.
\end{abstract}

Keywords: social abilities, pre-school children, poverty.

\section{INTRODUCCIÓN}

Las habilidades sociales constituyen un amplio campo de investigación, con importantes desarrollos teóricos y metodológicos en las últimas décadas, y han despertado gran interés en diferentes áreas de la Psicología por sus aplicaciones.

Las destrezas sociales son una parte esencial de la actividad humana ya que el discurrir de la vida está determinado, al menor parcialmente, por el rango de las habilidades sociales (Caballo, 2005). Distintos estudios señalan que las habilidades sociales inciden en la autoestima, en la adopción de roles, en la autorregulación del comportamiento y en el rendimiento académico, entre otros aspectos, tanto en la infancia como en la vida adulta (Gil Rodríguez, León Rubio \& Jarana Expósito, 1995; Kennedy, 1992; Monjas Casares, 2002; Ovejero Bernal, 1998).

Se considera que las habilidades sociales son un medio excepcional de protección y promoción de la salud ya que los comportamientos sociales positivos favorecen la adaptación, la aceptación de los otros, los refuerzos positivos y el bienestar del sujeto (Crespo Rica, 2006; Prieto Ursua, 2000).

Correspondencia: Ana Betina Lacunza. Consejo Nacional de Investigaciones Científicas y Técnicas (CONICET). Fac. de Ciencias de la Salud, Universidad del Norte Santo Tomás de Aquino, Argentina. Correo Electrónico: anabetinalacunza@ciudad.com.ar. 
Los comportamientos sociales se aprenden a lo largo del ciclo vital, por lo que ciertas conductas de los niños para relacionarse con sus pares, ser amable con los adultos o reaccionar agresivamente, entre otras, depende del proceso de socialización. Diferentes enfoques evolutivos coinciden en señalar que este proceso se inicia con el nacimiento del niño. Por ejemplo, desde una perspectiva conductista se han encontrado indicios de una interacción social primaria ya en los primeros meses de vida. El estudio de Kaye (1982) ha demostrado que durante el amamantamiento se configura una especie de diálogo entre el comportamiento de la madre y el niño; las madres tienden a interactuar con sus hijos siguiendo un modelo actividad-pausa de la succión. Esta evidencia señala que la base de la interacción social primaria se produce en relación a dos factores: a) el comportamiento espontáneo del niño, organizado temporalmente según mecanismos endógenos y b) la sensibilidad de la madre para esta periodicidad y su disponibilidad para adaptarse a este modelo (Schaffer, 1990).

Por su parte, las investigaciones longitudinales de Dunn (1988) en niños británicos muestran que la comprensión social de aquellos se inicia antes de la adquisición plena del lenguaje. Esta autora trata de mostrar cómo los niños van conformando capacidades cognitivas que hacen posible experimentar y comprender posteriormente las situaciones sociales. A los dos o tres años de vida, el niño vivencia una serie de situaciones que le permite organizar el mundo social y comprender normas, prohibiciones tanto como la expresión de sus propios derechos. Esto lo lleva a percibirse a si mismo y a los otros y, por lo tanto, a adquirir una competencia social (Delval, 1994).

Estos estudios evolutivos destacan cómo la socialización de un niño en sus primeros años de vida se produce gracias a la interrelación de factores biológicos, cognitivos y emocionales. Respecto al factor biológico cabe destacar la estructura corpórea (por ejemplo, el aparato oral, los órganos de los sentidos, entre otros) y funcional que facilitan la utilización de dichas estructuras. Ahora bien, el uso de estas estructuras está regulado por la sensibilidad materna ante el comportamiento del niño. Por otro lado, los factores cognitivos son necesarios para el comportamiento social, ya que la memoria y las reestructuraciones cognitivas logradas hacia fines del primer año de vida tienen efecto en el comportamiento social.
Entre estos desarrollos cognitivos se encuentran la capacidad de superar la situación inmediata, la capacidad para considerar varios acontecimientos simultáneamente, la diferenciación medio-fin y una mayor flexibilidad en el despliegue de la atención, principalmente a partir del cuatro y quinto estadio del desarrollo sensoriomotriz (Oiberman, Mansilla \& Orellana, 2002; Piaget \& Inhelder, 2000/ 1969). A esto se agrega el lenguaje verbal, más desarrollado a los dos años. Entre los aspectos emocionales se destaca la conformación de una conducta de apego, puesto que distintos autores (Ainsworth, 1974; Bowlby, 1983, 1986, 1989, Casullo \& Fernández Liporace, 2005) afirman que la internalización de experiencias vinculares dan lugar a modelos de comportamiento psicosocial, puesto que se ha comprobado, siempre y cuando el desarrollo evolutivo sea normal, que la conducta de apego lleva al establecimiento de vínculos afectivos entre el niño y su cuidador, en un principio, como entre los adultos, tiempo más tarde. El apego tiene una función adaptativa para el niño, los padres y el grupo familiar, como así también para la especie. Desde una visión objetiva, su sentido último es favorecer la supervivencia, manteniendo próximos y en contacto a las crías y a los progenitores (o quienes cumplan esta función), ya que estos son los que protegen y ofrecen los cuidados durante la infancia. Por otro lado, desde lo subjetivo, la función del apego va a proporcionar una seguridad emocional. El niño quiere a las figuras de apego porque con ellas se siente seguro, aceptado incondicionalmente, protegido y con los recursos emocionales y sociales necesarios para su bienestar (Ortiz, Fuentes \& López, 1999).

En síntesis, el proceso de socialización desplegado desde la temprana infancia posibilita en el niño el conocimiento de pautas, reglas, prohibiciones, entre otros, la conformación de los vínculos afectivos, la adquisición de comportamientos socialmente aceptables y la participación de los otros en la construcción de su personalidad (López \& Fuentes Rebollo, 1994).

De allí que resulte esencial identificar cómo aparecen los comportamientos sociales en los años preescolares, puesto que el aprendizaje y práctica de habilidades sociales posibilita la adaptación del niño a diferentes grupos y contextos.

La elección de niños pertenecientes a contextos de pobreza obedece a que se trata de una población vulnerable. 
Diversos autores, desde la Psicología, han dedicado sus investigaciones a determinar cuánto y cómo la falta de acceso a los bienes de la cultura en un sentido amplio y la exclusión social que ello implica afecta las capacidades psicológicas del niño. Restringe considerablemente la posibilidad del sujeto en crecimiento de aprehender su realidad más próxima y de hacer aprendizajes significativos que aseguren calidad de vida e inclusión en un grupo.

Sin embargo, el valor de este estudio es haber identificado no sólo los déficits sino las potencialidades de niños preescolares insertos en contextos de pobreza. Estos recursos potenciales -las habilidades sociales- pueden considerarse un factor protector de la salud, en la medida que actúen como un amortiguador del impacto de estímulos estresantes derivados de la pobreza.

\section{LAS HABILIDADES SOCIALES EN LOS AÑOS PREESCOLARES}

Monjas Casares (2002) señala que una tarea evolutiva esencial del niño es la de relacionarse adecuadamente con pares y adultos, conformando vínculos interpersonales. Para ello es necesario que éste adquiera, practique e incluya en su comportamiento una serie de capacidades sociales que le permitan un ajuste a su entorno más próximo.

Estas capacidades se denominan habilidades sociales y se definen como un conjunto de conductas que permiten al individuo desarrollarse en un contexto individual o interpersonal expresando sentimientos, actitudes, deseos, opiniones o derechos de un modo adecuado a la situación (Caballo, 1993). Generalmente, posibilitan la resolución de los problemas inmediatos como la probabilidad de reducir problemas futuros en la medida que el individuo respeta las conductas de los otros.

Las habilidades sociales son un conjunto de comportamientos aprendidos que incluyen aspectos conductuales, cognitivos y afectivos. La característica esencial de estas habilidades es que se adquieren principalmente a través del aprendizaje por lo que no pueden considerárselas un rasgo de personalidad. No obstante, investigaciones españolas (Garaigordobil \& García de Galdeano, 2006; Pérez Fernández \& Garaigordobil Landazabal, 2004) destacan conexiones estrechas entre comportamientos empáticos -habilidades sociales positivas- $y$ aspectos estrucutrales de la personalidad, como es el autoconcepto.

Tal como se planteó, el aprendizaje de las habilidades sociales se inicia desde el nacimiento a través del proceso de socialización. Diversos enfoques evolutivos coinciden en señalar que en los años preescolares o de niñez temprana (Papalia, Wendkos Olds \& Feldman, 2001) las adquisiciones motrices y mentales favorecen el desarrollo social y emocional del niño, por lo que la enseñanza y práctica de habilidades sociales resulta fundamental. Alrededor de los 3 años, los niños adelgazan y crecen tanto el tronco como las piernas y los brazos, aunque la cabeza es relativamente más grande que otras partes del cuerpo, las que a su vez, siguen su crecimiento tomando cada vez más el aspecto de un adulto (Papalía et al., 2001). Esto posibilita que deje de ser mirado como un bebé y pueda separarse del vínculo simbiótico psicológico con la madre, con el afianzamiento de los vínculos fraternos y el descubrimiento de la relación paterna (Griffa \& Moreno, 2005). Es un periodo en el que el juego ocupa un lugar clave para la socialización, ya que el niño está volcado al mundo exterior, aunque todavía mantiene una actitud cognitiva egocéntrica.

El periodo de los tres a los cinco años es fundamental para el desarrollo de las capacidades sociales, ya que el niño vivencia y registra una serie de situaciones que le permiten organizar su mundo social, comprender normas y prohibiciones como expresar sus propios derechos. Esto permite que el niño pueda percibirse a si mismo y a los otros de un modo más integrado, adquiriendo una competencia social.

Durante este periodo, el niño efectúa una transición desde el juego paralelo hacia un juego más interactivo y cooperativo, por lo que las interacciones con otros niños suelen ser más frecuentes y duraderas. Surge así la necesidad del niño de desarrollar habilidades para resolver exitosamente conflictos con pares o poder jugar con niños desconocidos. Según Gottman (1983) estas habilidades son predictoras de la capacidad del niño para hacer nuevas amistades.

Desde la perspectiva socio-cognitiva, autores como Flavell (1993), Wellman (1990), entre otros, sostienen que alrededor de los tres años, el niño puede comprender la mente de los otros y distinguirla respecto al mundo material. Estos avances del pensamiento son fundamentales en las habilidades sociales, particularmente en el desarrollo de la empatía. 
Además, las relaciones con los otros suelen ir asociadas a expresiones emocionales por lo que, sobre todo después de los dos años, los niños son capaces de reconocer sus propios estados emotivos como también de los demás.

En este periodo se acrecientan las interacciones sociales del niño, sobre todo por su ingreso al ámbito escolar. La vinculación con los otros le posibilita no sólo el manejo de las emociones sino la internalización de pautas y roles, elementos esenciales para un desenvolvimiento eficaz en periodos posteriores. Además, el niño paulatinamente va conformando modelos sobre el funcionamiento mental de las otras personas, estableciendo así una especie de causalidad psicológica sobre las relaciones sociales (Delval, 1994).

Si bien las investigaciones han encontrado sólidas relaciones entre las competencias sociales y el funcionamiento psicológico, la mayoría de los estudios centraron su interés en población adulta (véase Caballo, 1993; De Miguel \& Pelechano Barberá, 1996; Ovejero Bernal, 1998). Sólo en los últimos años surgieron investigaciones respecto al estudio de las habilidades sociales en la infancia y adolescencia, particularmente con población en edad escolar (Ison, 1997; Michelson, Sugai, Wood \& Kazdin, 1983; Monjas Casares, González Moreno \& col., 1998).

Todavía son escasos los autores que han trabajado con población infantil, particularmente preescolar, sobre el desarrollo de las habilidades sociales. Por ejemplo, Álvarez, Álvarez-Monteserín, Cañas, Jiménez \& Petit (1990) sostienen que las habilidades sociales básicas para niños preescolares (tres a cinco años) incluyen aquellas vinculadas a la interacción con el juego, la expresión de emociones, la autoafirmación y la conversación. Estos autores españoles han diseñado un programa para el desarrollo de tales habilidades con el objetivo de que los niños aprendan a ser críticos, libres, responsables y solidarios.

Por su parte, Merrell (2002) considera a las habilidades sociales como conductas positivas o de adaptación que permiten el óptimo desarrollo personal y social de niños preescolares. En su escala Preschool and Kindergarten Behavior Scales (PKBS-2) las operacionaliza en cooperación social, interacción social e independencia social.

Puede afirmarse que la insuficiencia de estudios empíricos en población preescolar podría deberse no sólo a las dificultades intrínsecas a la definición del constructo habilidad social, tal como planteara Fernández Ballesteros (1994), sino también a la complejidad metodológica que exige la evaluación de dichas competencias en la infancia (Forns i Santacana, 1993; Gresham, 1988; Maganto Mateo \& Cruz Saez, 2001; Sendín, 2000), ya que el énfasis debe estar puesto en una perspectiva múltimétodo y multicontexto.

\section{OBJETIVOS}

- Describir una serie de habilidades sociales en niños de 3 a 5 años de S.M. de Tucumán (Argentina) según el sexo y los niveles de pobreza. - Analizar la cualidad de las habilidades sociales más recurrentes en este grupo.

\section{MÉTODO}

Participantes: 318 padres y/o cuidadores de niños, entre 3 y 5 años, asistentes a Centros de Atención Primaria de la Salud (CAPS) de San Miguel de Tucumán (Argentina). Todos los participantes pertenecían a contextos de pobreza.

A nivel operacional, dos variables definieron a la pobreza: el nivel educativo alcanzado y la ocupación actual de ambos padres del niño. La inclusión de los datos de la madre, y no solo del padre (generalmente el jefe del hogar y principal sostén) obedece a la necesidad de describir aquellas características maternas que se relacionen con el proceso de desarrollo infantil. De la combinación de ambas variables se ha establecido un nivel mayor y otro menor de pobreza.

El nivel de menor pobreza incluyó a aquellos padres con una escolaridad superior a primaria completa y con ocupaciones estables de baja calificación. Por su parte, el nivel mayor incluyó a aquellos progenitores que presentaran un nivel educativo mínimo y ocupaciones inestables o sólo ligadas a planes sociales.

La determinación de tales categorías se debió a la gran heterogeneidad de los datos obtenidos en la encuesta sociodemográfica. Este método dio lugar a una categorización abreviada del NES bajo.

Se encontró que el $58 \%$ de los niños y sus familias incluidas en este estudio presentaron un nivel mayor de pobreza, mientras que el $42 \%$ restante de los niños presentaba un menor nivel de pobreza (padres que habían completado sus estudios primarios y desempeñaban ocupaciones estables de baja calificación) (véase tabla 1). 


\begin{tabular}{|c|c|c|c|c|c|}
\hline \multirow{3}{*}{ Edad } & \multicolumn{4}{|c|}{ NES bajo } & \multirow{3}{*}{ Totales $(\mathrm{N})$} \\
\hline & \multicolumn{2}{|c|}{$\begin{array}{l}\text { Menor } \\
\text { pobreza }\end{array}$} & \multicolumn{2}{|c|}{$\begin{array}{l}\text { Mayor } \\
\text { pobreza }\end{array}$} & \\
\hline & $f$ & $\%$ & $f$ & $\%$ & \\
\hline 3 años & 24 & 25 & 72 & 75 & 96 \\
\hline 4 años & 38 & 37 & 64 & 63 & 102 \\
\hline 5 años & 71 & 59 & 49 & 41 & 120 \\
\hline
\end{tabular}

Tabla 1. Nivel Socioeconómico bajo según edad $(\mathrm{N}=318)$

\section{INSTRUMENTOS}

- Escala de Habilidades Sociales (Lacunza, 2005): diseñada para evaluar las habilidades sociales a partir de la percepción de padres y/o cuidadores del niño. Se tratan de escalas con tres opciones de respuesta, cuyo puntaje total discrimina un alto o bajo nivel de habilidades sociales. La escala para 3 años está integrada por 12 ítems (fiabilidad global de .72) como así también la de 4 años (fiabilidad global de .77), en tanto, la de 5 años tiene también 16 ítems (fiabilidad global de .85) (Lacunza, Castro Solano \& Contini, 2008). Los instrumentos han sido validados con población perteneciente a contextos de pobreza (Lacunza, 2007).

- Encuesta sociodemográfica (diseñada para el presente estudio).

\section{PROCEDIMIENTO}

Los padres de los niños participantes respondieron las escalas de habilidades sociales y la encuesta sociodemográfica en los CAPS cuando realizaban el control pediátrico de sus hijos.

La aplicación fue individual con un tiempo aproximado de 10 minutos. En el presente trabajo sólo se consideraron de la encuesta sociodemográfica aquellas variables vinculadas al nivel socioeconómico (NES).

Se realizó un análisis cuantitativo de los instrumentos según la franja de edad, discriminándose el puntaje total de la escala y las diferencias entre cada grupo evaluado (varonesmujeres y niños de menor-mayor pobreza) a partir de un análisis univariado de la varianza (ANOVA). Posteriormente se establecieron las frecuencias de los ítems de las escalas.

Los análisis fueron realizados con el paquete estadístico SPSS-11.5.

\section{RESULTADOS}

El primer objetivo fue describir una serie de habilidades sociales en niños de 3 a 5 años de S.M. de Tucumán (Argentina) en situación de pobreza.

En el grupo de niños de tres años el puntaje máximo de la escala fue de 36 puntos. Se encontró que, según la percepción parental, las niñas de menor y mayor pobreza presentaban más habilidades sociales respecto a sus pares varones (véase tabla 2). Los análisis univariados no mostraron diferencias estadísticamente significativas con relación al sexo de los niños participantes $(F(1,50.15)=1.99, p>0.05) y$ el nivel socioeconómico bajo $(F(1,93.47)=$ $3.71, p>0.05)$. Tampoco se observó interacción entre el sexo y el nivel socioeconómico $(F(1,11.65)=0.46, n s)$ ya que se encontró que la variabilidad total se debió fundamentalmente a la variabilidad dentro de los grupos.

\begin{tabular}{|c|c|c|c|c|}
\hline \multirow{2}{*}{ Sexo } & \multicolumn{4}{|c|}{ Nes bajo } \\
\cline { 2 - 5 } & \multicolumn{2}{|c|}{$\begin{array}{c}\text { Menor } \\
\text { pobreza }\end{array}$} & \multicolumn{2}{c|}{$\begin{array}{c}\text { Mayor } \\
\text { pobreza }\end{array}$} \\
\cline { 2 - 5 } & $\mathrm{M}$ & $\mathrm{DE}$ & $\mathrm{M}$ & $\mathrm{DE}$ \\
\hline Varón & 29,76 & 3,24 & 26,67 & 6,04 \\
\hline Mujer & 30,63 & 2,97 & 29,15 & 4,91 \\
\hline
\end{tabular}

Tabla 2. Descriptivos de puntajes en Escala de habilidades sociales según sexo y niveles de pobreza. Grupo de niños de 3 años.

En el grupo de niños de cuatro años el puntaje máximo de la escala también fue de 36 puntos. Se observó que las niñas de menor pobreza obtuvieron puntajes mas altos en la prueba, por lo que su repertorio de habilidades sociales era mayor respecto a sus pares (véase tabla 3). Los análisis univariados no mostraron diferencias estadísticamente significativas con relación al sexo de los niños participantes $(F(1,2.27)=$ $0.08, p>0.05)$ y el nivel socioeconómico bajo $(F(1,25)=0.87, p>0.05)$. Tampoco se observó interacción entre el sexo y el nivel socioeconómico $(F(1,69.24)=2.42$, ns) ya que se encontró que la variabilidad total se debió fundamentalmente a la variabilidad dentro de los grupos.

En el grupo de niños de cinco años se mantuvo una tendencia similar respecto a sus pares de cuatro años, ya que la niñas con un menor nivel de pobreza presentaron las puntuaciones más altas en la escala ( $\mathrm{M}=38.96, \mathrm{DE}=7.06)$ (ver tabla 4), aunque ninguno de los grupos alcanzó el puntaje máximo de la prueba (48 puntos).

\begin{tabular}{|c|c|c|c|c|c|}
\hline \multirow{3}{*}{ Sexo } & \multicolumn{4}{|c|}{ Nes bajo } & \multirow{3}{*}{$\begin{array}{l}\text { Tabla 3. Descrip- } \\
\text { tivos de puntajes } \\
\text { en Escala de ha- } \\
\text { bilidades sociales } \\
\text { según sexo y ni- }\end{array}$} \\
\hline & \multicolumn{2}{|c|}{$\begin{array}{l}\text { Menor } \\
\text { pobreza }\end{array}$} & \multicolumn{2}{|c|}{$\begin{array}{l}\text { Mayor } \\
\text { pobreza }\end{array}$} & \\
\hline & $M$ & $\mathrm{DE}$ & $\mathrm{M}$ & $\mathrm{DE}$ & \\
\hline Varón & 29,04 & 4,23 & 28,57 & 5,78 & 然 \\
\hline Mujer & 30 & 5,28 & 27,2 & 6,48 & s \\
\hline
\end{tabular}


Los análisis univariados no mostraron diferencias estadísticamente significativas con relación al sexo de los niños participantes $(F(1,2.64)=0.04, p>0.05)$ y el nivel socioeconómico bajo $(F(1,59.25)=0.97$, $p>0.05)$. Tampoco se observó interacción entre el sexo y el nivel socioeconómico ( $F$ $(1,78.73)=1.29$, ns) ya que se encontró que la variabilidad total se debió fundamentalmente a la variabilidad dentro de los grupos.

El segundo objetivo fue analizar la cualidad de las habilidades sociales más recurrentes en este grupo. En el grupo de niños de 3 años la opción de respuesta frecuentemente fue la más recurrente, excepto en el ítem 4: En sus relaciones con otros niños dice gracias, donde la mayor recurrencia fue para la opción nunca, elección que obedeció a ciertas características culturales de la muestra. En tanto, el $40 \%$ de los informantes refirieron que sus hijos no solían acercarse a otros niños del mismo sexo para iniciar juegos o conversar (ítem 6) (ver tabla 5).

\begin{tabular}{|c|c|c|c|c|}
\hline \multirow{2}{*}{ Sexo } & \multicolumn{4}{|c|}{ Nes bajo } \\
\cline { 2 - 5 } & \multicolumn{2}{|c|}{$\begin{array}{c}\text { Menor } \\
\text { pobreza }\end{array}$} & \multicolumn{2}{c|}{$\begin{array}{c}\text { Mayor } \\
\text { pobreza }\end{array}$} \\
\cline { 2 - 5 } & $\mathrm{M}$ & $\mathrm{DE}$ & $\mathrm{M}$ & $\mathrm{DE}$ \\
\hline Varón & 37.2 & 8,2 & 37 & 8,27 \\
\hline Mujer & 30 & 5,28 & 27,2 & 6,48 \\
\hline
\end{tabular}

Tabla 4. Descriptivos de puntajes en Escala de habilidades sociales según sexo y niveles de pobreza. Grupo de niños de 5 años.

\begin{tabular}{|c|c|c|c|}
\hline \multirow{2}{*}{$\begin{array}{l}\text { Escala de habilidades } \\
\text { sociales }\end{array}$} & \multicolumn{3}{|c|}{ Opciones de respuesta (\% de presencia) } \\
\hline & Nunca & $\begin{array}{l}\text { Algunas } \\
\text { veces }\end{array}$ & $\begin{array}{l}\text { Frecuentem } \\
\text { ente }\end{array}$ \\
\hline $\begin{array}{l}\text { 1. Sonríe y/o saluda a las } \\
\text { personas que conoce } \\
\text { (familiares, vecinos) }\end{array}$ & 17,7 & 5,2 & 77,1 \\
\hline $\begin{array}{l}\text { 2. Puede decir su nombre } \\
\text { cuando se lo preguntan }\end{array}$ & 12,5 & 12,5 & 75 \\
\hline $\begin{array}{l}\text { 3. Comparte algún alimento, } \\
\text { juguete u otra pertenencia } \\
\text { con un niño que conoce }\end{array}$ & 12,5 & 12,5 & 75 \\
\hline $\begin{array}{l}\text { 4. En sus relaciones con otros } \\
\text { niños dice "gracias" }\end{array}$ & 42,7 & 16,7 & 40,6 \\
\hline $\begin{array}{l}\text { 5. Menciona una aprobación } \\
\text { cuando un niño hace algo } \\
\text { de su agrado }\end{array}$ & 33,3 & 4,2 & 62,5 \\
\hline $\begin{array}{l}\text { 6. Se acerca a otros niños del } \\
\text { mismo sexo para iniciar } \\
\text { juegos o conversar }\end{array}$ & 40,6 & 8,3 & 51 \\
\hline $\begin{array}{l}\text { 7. Se adapta a los juegos y/o } \\
\text { actividades que otros niños } \\
\text { ya están haciendo }\end{array}$ & 37,5 & 7,3 & 55,2 \\
\hline $\begin{array}{l}\text { 8. Se queja si un niño le hace } \\
\text { algo desagradable }\end{array}$ & 11,5 & 4,2 & 84,4 \\
\hline $\begin{array}{l}\text { 9. Dice "gracias" en sus } \\
\text { relaciones con sus padres }\end{array}$ & 30,2 & 16,7 & 53,1 \\
\hline $\begin{array}{l}\text { 10. Menciona halagos para sus } \\
\text { padres o a alguno de ellos }\end{array}$ & 31,3 & 5,2 & 63,5 \\
\hline $\begin{array}{l}\text { 11. Puede responder } \begin{array}{l}\text { a una } \\
\text { pregunta sencilla de un } \\
\text { adulto }\end{array} \\
\end{array}$ & 25 & 8,3 & 66,7 \\
\hline $\begin{array}{l}\text { 12. Pregunta a los adultos } \\
\text { sobre el porqué de algunas } \\
\text { situaciones }\end{array}$ & 24 & 8,3 & 67,7 \\
\hline
\end{tabular}

Tabla 5. Frecuencias de opciones de respuesta, escala de habilidades sociales. Grupo de niños de 3 años.
En el grupo de de niños de 4 años la opción de respuesta frecuentemente también fue la más recurrente, menos en el ítem 3 (se presenta espontáneamente a otros niños), donde el $54 \%$ de los padres eligieron la opción de respuesta nunca. Por su parte, el $23 \%$ de los niños sólo algunas veces era amable con sus padres o cuidadores, según la percepción de éstos (ítem 9) (ver tabla 6). También en el grupo de niños de 5 años la opción de respuesta frecuentemente fue la más recurrente.

Cabe destacar que el $50 \%$ de los niños participantes no solía presentarse espontáneamente a otros niños y adultos (ítem 3) ni ser uno de los primeros en acercarse a otros niños que no conoce (ítem 6). Las dificultades en la interacción con pares se vio reflejada en las repuestas a los ítems 8,9 y 10, ya que más del $30 \%$ de los niños no empleaba habilidades vinculadas al dialogo y acercamiento a otros niños (ver tabla 7).

\begin{tabular}{|l|c|c|c|}
\hline \multirow{2}{*}{ Escala de habilidades sociales } & \multicolumn{3}{|c|}{ Opciones de respuesta (\% de } \\
presencia)
\end{tabular}

Tabla 6. Frecuencias de opciones de respuesta, escala de habilidades sociales. Grupo de niños de 4 años.

\section{DISCUSIÓN}

Este trabajo tuvo como objetivos describir una serie de habilidades sociales en niños de 3 a 5 años de San Miguel de Tucumán (Argentina) en situación de pobreza y analizar la cualidad de las habilidades sociales más recurrentes. 


\begin{tabular}{|c|c|c|c|}
\hline \multirow{2}{*}{\begin{tabular}{|c|} 
Escala de habilidades \\
sociales
\end{tabular}} & \multicolumn{3}{|c|}{ Opciones de respuesta (\% de presencia) } \\
\hline & Nunca & $\begin{array}{l}\text { Algunas } \\
\text { veces }\end{array}$ & $\begin{array}{l}\begin{array}{c}\text { Frecuentem } \\
\text { ente }\end{array} \\
\end{array}$ \\
\hline $\begin{array}{l}\text { 1. Sonríe y responde a } \\
\text { otras personas cuando } \\
\text { es oportuno }\end{array}$ & 19,2 & 14,2 & 66,7 \\
\hline $\begin{array}{l}\text { 2. Saluda y se despide de } \\
\text { las personas de un lugar } \\
\text { conocido }\end{array}$ & 28,3 & 17,5 & 54,2 \\
\hline $\begin{array}{l}\text { 3. Se presenta } \\
\text { espontáneamente a otros } \\
\text { niños y adultos }\end{array}$ & 50 & 5,8 & 44,2 \\
\hline $\begin{array}{l}\text { 4. Hace "pequeños favores" } \\
\text { a otros niños }\end{array}$ & 26,7 & 15,8 & 57,5 \\
\hline $\begin{array}{l}\text { 5. Con otros niños muestra } \\
\text { conductas de cortesía } \\
\text { (utiliza frases como: "por } \\
\text { favor", "gracias", } \\
\text { "perdón") } \\
\end{array}$ & 32,5 & 20 & 47,5 \\
\hline $\begin{array}{l}\text { 6. Suele ser uno de los } \\
\text { primeros en acercarse a } \\
\text { otros niños que no } \\
\text { conoce }\end{array}$ & 50,8 & 5,8 & 43,3 \\
\hline $\begin{array}{l}\text { 7. Se une al juego de otros } \\
\text { niños }\end{array}$ & 32,5 & 10 & 57,5 \\
\hline $\begin{array}{l}\text { 8. Ayuda a un } \\
\text { cuando amigo } \\
\begin{array}{l}\text { dificultades } \\
\text { está }\end{array} \\
\text { en }\end{array}$ & 35 & 5,8 & 59,2 \\
\hline $\begin{array}{l}\text { 9. Ayuda a otros niños en } \\
\text { actividades y juegos }\end{array}$ & 33,3 & 9,2 & 57,5 \\
\hline $\begin{array}{l}\text { 10. Inicia una conversación } \\
\text { simple con otros niños }\end{array}$ & 31,7 & 10 & 58,3 \\
\hline $\begin{array}{l}\text { 11. Cuando abandona a un } \\
\text { grupo de niños los saluda } \\
\text { cordialmente }\end{array}$ & 26,7 & 9,2 & 64,2 \\
\hline $\begin{array}{l}\text { 12. Se une al diálogo y/o } \\
\text { actividades que } \\
\text { mantienen otros niños }\end{array}$ & 25,8 & 15 & 59,2 \\
\hline $\begin{array}{l}\text { 13. Participa activamente en } \\
\text { actividades } \\
\text { conversaciones con otros } \\
\text { niños }\end{array}$ & 22,5 & 15 & 62,5 \\
\hline $\begin{array}{l}\text { 14. Es amable con los } \\
\text { adultos conocidos }\end{array}$ & 16,7 & 6,7 & 76,7 \\
\hline $\begin{array}{l}\text { 15. Menciona halagos para } \\
\text { sus padres } \\
\text { adultos } \\
\text { atros } \\
\text { conocidos }\end{array}$ & 10,8 & 6,7 & 82,5 \\
\hline
\end{tabular}

Tabla 7. Frecuencias de opciones de respuesta, escala de habilidades sociales. Grupo de niños de 5 años

Los resultados mostraron que tanto niños y niñas, de contextos de pobreza, cuentan con habilidades sociales para afrontar situaciones cotidianas, ya que no se observaron diferencias estadísticamente significativas en las puntuaciones del instrumento diseñado para este estudio. A pesar de la adversidad asociada a la pobreza, estos niños han logrado adquirir una serie de habilidades sociales, tales como saludar, mencionar su nombre, adaptarse a los juegos de otros niños, halagar a sus padres, denunciar cuando otro niño le hace algo desagradable, iniciativa para vincularse con pares no conocidos, comportamientos cooperativos, expresión de sentimientos positivos en sus interacciones con adultos, entre otros.

Las habilidades sociales entre los tres y los cinco años implican la interacción con los pares, las manifestaciones prosociales, la exploración de reglas, la comprensión de las emociones, entre otros indicadores. Estos han sido relevantes en diversos estudios sobre comportamientos sociales infantiles (David \&
Murphy, 2007; Garner, Robertson \& Smith, 1997; Larsen, To \& Fireman, 2007).

En los niños preescolares, las habilidades sociales dan cuenta de un aprendizaje de pautas y modelos que le permiten relacionarse con los otros de una forma efectiva y satisfactoria.

A los tres años aproximadamente, el niño va adquiriendo habilidades ligadas a la interacción con su grupo primario (padres y hermanos) y con pares. Estas habilidades se observaron en indicadores como: menciona una aprobación cuando un niño hace algo de su agrado (ítem 5), se acerca a otros niños del mismo sexo para iniciar juegos o conversar (ítem 6), se adapta a los juegos y/o actividades que otros niños ya están haciendo (ítem 7), los que fueron descriptos como comportamientos recurrentes en este grupo de niños. Hartup (1992) sostiene que la relación del niño con otros pequeños contribuye tanto a su desarrollo social como cognitivo; las relaciones de amistad son comprendidas desde la temprana infancia ya que los pares sirven como recursos emocionales al actuar como soportes ante acontecimientos vivenciados como negativos para el niño (por ejemplo, el ingreso al ámbito escolar o a la guardería maternal).

Este autor sostiene que los pares también actúan como recursos cognitivos, ya que ellos transmiten el sentido de la cooperación y la colaboración. Así, estas primeras relaciones pueden actuar como modelos para las subsiguientes. Si bien, a los tres años el niño mantiene un pensamiento egocéntrico, lo que se manifiesta también en sus juegos, la utilización de la ficción para comprender la realidad, el como sí propio de este tipo de juegos le permite al niño el desarrollo del área social e intelectual (Berk \& Winsler, 1995). Aunque el egocentrismo puede continuar hasta los cinco o los seis años, ya a los cuatro años, los comportamientos cooperativos suelen ser más constantes en la medida que estas habilidades sean valoradas en su contexto más próximo. No debe olvidarse que la socialización no sólo implica la interacción con otros sino que es un proceso progresivo de asimilación de actitudes, valores y costumbres de una sociedad, por lo que el niño aprende qué comportamientos son aceptables dentro de su grupo (Hoffman, Paris \& Hall, 1995).

A los cuatro años, la cooperación se expresa en el cumplimiento de normas y ayuda a pares o adultos, entre otros observables. 
En la presente investigación aparecieron como muy frecuentemente practicados ciertos comportamientos cooperativos (ítem 4: pregunta a otros niños si puede ayudarlos en actividades e ítem 9: es amable con sus padres y otros adultos conocidos), resultados que coinciden con los estudios de McClelland \& Morrison (2003) y Nabors, Willoughby \& Badawi (1999) en niños preescolares y con necesidades educativas especiales. A los cinco años, los indicios de la cooperación son más constantes ya que el niño ha adquirido conductas prosociales tales como ayudar o compartir; éstas aparecieron como frecuentes en el presente estudio, sobre todo en la respuesta a indicadores como: hace "pequeños favores" a otros niños (ítem 4), ayuda a un amigo cuando está en dificultades (ítem 8), ayuda a otros niños en actividades y juegos (ítem 9), es amable con los adultos conocidos (ítem 14).

Las habilidades sociales están relacionadas con la expresión y comprensión de estados emocionales, todo lo cual le permite al niño preescolar tener un control respecto a cómo demostrar sus sentimientos y cómo responder ante los sentimientos de los demás, principalmente su grupo familiar (Garner \& Power, 1996). Los niños participantes mostraron alta capacidad de expresar sentimientos negativos (por ejemplo, quejarse si un par le hace algo desagradable) o manifestar emociones positivas hacia sus padres (por ejemplo, halagar a sus padres o a alguno de ellos). Está comprobado que la expresión de tales comportamientos deriva de la influencia social y de las características de personalidad parentales (Roa Capilla \& Del Barrio Gándara, 2003).

Aquellos comportamientos sociales vinculados a la expresión de emociones positivas, principalmente de elogios hacia los adultos, aparecieron con una mayor frecuencia en todas las edades estudiadas. Las madres encuestadas refirieron habilidades de cortesía y amabilidad con el adulto, no sólo por responder a preguntas de personas mayores sino por utilizar palabras y gestos adecuados a las figuras parentales.

Los resultados mostraron la ausencia de diferencias estadísticas en el empleo de las habilidades sociales según el sexo, aunque algunos estudios empíricos (Merrell \& Gimpell, 1998; Navarro, 2004) señalan que existe un patrón de comportamiento femenino y otro masculino de relaciones interpersonales tanto en la infancia como en la adolescencia.
Si bien no se encontraron diferencias estadísticas respecto al nivel de habilidades sociales según los niveles de NES bajo, el contexto es clave para el aprendizaje de las habilidades sociales. El aprendizaje de las habilidades sociales está muy vinculado a las características del entorno como a la relevancia que presenten ciertos comportamientos sociales considerados exitosos, por lo que la cultura contribuye en este sentido (Gardner, 1994).

Según Caballo (2005), la adaptación y participación en diferentes contextos, está determinada, al menos parcialmente, por el tipo de habilidades sociales. Sin embargo, Monjas Casares (2002) considera que la sola presencia de comportamientos sociales no determina que un niño sea socialmente competente, sino que debe poner en juego este repertorio de habilidades sociales en una situación específica y ser valorado positivamente, para que su actuación sea competente. De allí, que no es más hábil el niño que más conductas posea, sino el que sea capaz de percibir y discriminar las señales del contexto y elegir una combinación de comportamientos adecuados para esa situación determinada. Aquí entran en juego recursos internos del niño, particularmente cognitivos y afectivos, los que a su vez, se desarrollan con recursos externos vinculados a la interacción con pares y adultos, particularmente los estilos de crianza y los sistemas de valores asociados a la cultura parental.

En definitiva, los niños participantes desde la percepción de sus padres, mostraron comportamientos sociales necesarios para el desenvolvimiento en su vida diaria, los que les permitían un ajuste psicológico en su contexto más próximo. Las relaciones sociales que establecían estos niños pueden considerarse como un factor protector de la salud, en la medida que el empleo de las habilidades sociales positivas contribuya al funcionamiento adaptativo.

Hay que recordar que el niño en situación de pobreza debe enfrentar un ambiente caracterizado por la incertidumbre y los estímulos estresantes. Si bien los recursos para afrontarlos suelen ser escasos, este estudio comprobó que las habilidades sociales positivas son capacidades que permiten la adaptación activa y, por lo tanto, pueden actuar como un amortiguador de los efectos negativos propios de la pobreza y la desigualdad social. 
Estos datos constituyen un paso previo para la formulación de programas de estimulación y potenciación de dichas capacidades sociales, las que operan como factores protectores de la salud.

\section{REFERENCIAS}

Ainsworth, M. (1974). The development of Infant- Mother Attachment. Review of Child Development. Chicago: Chicago Press.

Alvarez, A., Alvarez-Monteserín, M., Cañas, A., Jiménez, S. \& Petit, M. (1990). Desarrollo de las habilidades sociales en niños de 3-6 años. Guía práctica para padres y profesores. Madrid: Aprendizaje Visor.

Berk, L. \& Winsler, A. (1995). Scaffolding children's learning: Vygotsky and early childhood education. Washington, D. C.: National Association for the Education of Young Children.

Bowlby, J. (1983). La pérdida afectiva. Tristeza y depresión. Buenos Aires: Paidos.

Bowlby, J. (1986). Vínculos afectivos: formación, desarrollo y pérdida. Madrid: Morata.

Bowlby, J. (1989). Una base segura. Aplicaciones clínicas de una teoría del apego. Buenos Aires: Paidos.

Caballo, V. (1993). Relaciones entre diversas medidas conductuales y de autoinforme de las habilidades sociales. Psicología Conductual, 1, 73-99.

Caballo, V. (2005). Manual de Evaluación y entrenamiento de las habilidades sociales. ( $6^{\circ}$ Edición). Madrid: Siglo XXI Editores.

Casullo, M. \&Fernández Liporace, M. (2005). Los estilos de apego. Teoríaymedición. BuenosAires: JVEediciones.

Crespo Rica, S. (2006). Comportamiento social en la infancia. Trabajo presentado en el V Congreso Internacional "Educación y Sociedad". Disponible en: http:// congreso.codoli.org/area_3/Crespo-Rica3.pdf

David, K. \& Murphy, B. (2007). Interparental conflict and preschoolers' peer relations. The moderating roles of temperament and gender. Social development, 16 (1), 1-23.

De Miguel, A. \& Pelechano Barberá, V. (1996). Programa de entrenamiento en fases de solución de problemas para ancianos. En V. Pelechano Barberá (Coord.) (1996), Habilidades interpersonales: teoría mínima y programas de intervención (pp. 743-766). Vol III. Valencia: Promolibro.

Delval, J. (1994). El desarrollo humano. Madrid: Siglo XXI Editores.

Dunn, J. (1988). Los comienzos de la comprensión social. Buenos Aires: Nueva Visión.

Fernández Ballesteros, R. (1994). Evaluación conductual hoy. Un enfoque para el cambio en psicología clínica y de la salud. Madrid: Pirámide.
Flavell, J. (1993). El desarrollo cognitivo. Madrid: Visor. Forns i Santacana, M. (1993). Evaluación psicológica infantil. Barcelona: Barcanova.

Garaigordobil, M. \& García de Galdeano, P. (2006). Empatía en niños de 10 a 12 años. Psicothema, 18 (2), 180-186.

Gardner, H. (1994). Estructuras de la mente. La teoría de las inteligencias múltiples. Méjico: Fondo de Cultura Económica.

Garner, P. \& Power, T. (1996). Preschoolers Emotional Control in the Disappointment Paradigm and its relation to Temperament, Emotional Knowledge and Family Expressiveness. Child Development, 67 (4), 1406-1419.

Garner, P., Robertson, S. \& Smith, G. (1997). Preschool Children's Emotional Expressions with peers: the roles of gender and emotion socialization. Journal Sex Roles, 36 (11-12), 675-691.

Gil Rodríguez, F., León Rubio, J. \& Jarana Expósito, L. (Eds). (1995). Habilidades sociales y salud. Madrid: Pirámide.

Gottman, J..M. (1983). How children become friends. Monograph of the Society for Research in Child Development, 48 (3, Serial No. 108).

Gresham, F. (1988). Social skills: Conceptual and applied aspects of assessment, training and social validation. En J. Witt, S. Elliott \& F. Gresham (Comps.), Handbook of Behavior Therapy in Education, (pp. pp. 523546). Nueva York: Plenum Press.

Griffa, M. \& Moreno, J. (2005). Claves para una Psicología del Desarrollo. Vida prenatal. Etapas de la niñez. Buenos Aires: Lugar Editorial. Volumen 1.

Hartup, W. (1992). Having friends, making friends, and keeping friends: Relationships as educational contexts. ERIC Digest. Champaign, IL: ERIC Clearinghouse on Elementary and Early Childhood Education

Hoffman, L., Paris, S. \& Hall, E. (1995). Psicología del Desarrollo hoy. Madrid: Mc Graw-Hill. Volumen 1 (6 $6^{\circ}$ Edición).

Ison, M (1997). Déficits en habilidades sociales en niños con conductas problema. Revista Interamericana de Psicología, 3 (2), 243-255.

Kaye, K. (1982). The mental and social life of babies. Londres: Methuen.

Kennedy, J. (1992). Relationship of maternal beliefs and childrearing strategies to social competence in preschool children. Child Study Journal, 22 (1), 39-61.

Lacunza, A. (2005). Escala de Habilidades Sociales para niños de 5 años. Instrumento inédito.

Lacunza, A. (2007). Inteligencia y Desnutrición. La evaluación de las habilidades cognitivas y sociales en niños de Tucumán en contextos de pobreza. Tesis Doctoral. 
Lacunza, A., Castro Solano, A. \& Contini, N. (2008). Las habilidades sociales preescolares: construcción y validación de una escala para niños de contextos de pobreza. Revista de Psicología, Pontificia Universidad Católica del Perú (en prensa).

Larsen, J., To, Y. \& Fireman, G. (2007). Children's understanding and experience of mixed emotions. Psychological Science, 18 (2), 186-191.

López, F. \& Fuentes Rebollo, M. (1994). Revisión, análisis y clasificación de los estudios sobre desarrollo social. Infancia y Aprendizaje, 67-68, 163-185.

Maganto Mateo, C. \& Cruz Saez, S. (2001). Multitécnicas y multimétodos en la evaluación de la primera infancia. Revista Iberoamericana de Diagnóstico y Evaluación Psicológica, 12, (2), 149-181.

McClelland, M. \& Morrison, F. (2003). The emergent of learning- related social skills in preschool children. Early Childhood Research Quarterly, 18 (2), 206-224.

Merrell, K. \& Gimpel, G. (1998). Social skills of children and adolescents: Conceptualization, assessment, treatment. Mahwa, NJ: Lawrence Earlbaum Associates.

Merrell, K.W. (2002). Preschool and Kindergarten Behaviour Rating Scales (PKBS-2). Austin, TX: PRO-ED.

Michelson, L., Sugai, D., Wood, R. \& Kazdin, A. (1987). Las habilidades sociales en la infancia: Evaluación y tratamiento. Barcelona: Martínez Roca.

Monjas Casares, M. \& González Moreno, B. (1998). Las habilidades sociales en el curriculo (Serie Colección N 146). España: Centro de Investigación y Documentación Educativa- CIDE. España, № 146.

Monjas Casares, M. (2002). Programa de enseñanza de habilidades de interacción social (PEHIS) para niños y niñas en edad escolar. Madrid: CEPE.

Nabors, L., Willoughby, J. \& Badawi, M. (1999). Relations between Activities and Cooperative Playground Interactions for Preschool-Age Children with Special Needs. Journal of Developmental and Physical Disabilities, 11 (4), 339-352.

Navarro, E. (2004). Género y relaciones personales íntimas. En E. Barberá, y I. Martínez (Coord.), Psicología y Género (pp. 171-192). Madrid: Pearson Educación.
Oiberman, A. Mansilla, M. \& Orellana, L. (2002) Nacer y Pensar. Construcción de la Escala de Inteligencia Sensorio-motriz (EAIS) de 6 meses a 2 años. Vol.1 Buenos Aires: Ediciones CIIPME-CONICET.

Ortíz, M, Fuentes, M. \& López, F. (1999). Desarrollo socioafectivo en la primera infancia. En J. Palacios, A. Marchesi \& C. Coll (Comps.), Desarrollo psicológico y Educación (pp. 151-175). Madrid: Alianza ( $2^{\circ}$ edición).

Ovejero Bernal, A. (1998). Las habilidades sociales y su entrenamiento en el ámbito escolar. En Gil, F. \& León, J. (comp.), Habilidades sociales. Teoría, investigación e intervención (pp. 169-185). Madrid: Síntesis Psicológica.

Papalia, D, Wendkos Olds, S. \& Feldman, R. (2001) Psicología del desarrollo. Colombia: Mc Graw- Hill (8º edición).

Pérez Fernández, J. \& Garaigordobil Landazabal, M. (2004). Relaciones de la socialización con inteligencia, autoconcepto y otros rasgos de la personalidad en niños de 6 años. Apuntes de Psicología, 22 (2), 153-169.

Piaget, J. \& Inhelder, B. (2000). Psicología del niño. Madrid: Morata. Publicado por primera vez en 1969.

Prieto Ursua, M. (2000). Variables psicológicas relevantes en el estudio de los menores con trastornos psíquicos. Ponencia presentada en el I Congreso Hispano-Portugués de Psicología, 21 al 23 de setiembre, Santiago de Compostela. Disponible en: http://www. fedap.es/congreso_santiago/trabajos/mprieto2.htm.

Roa Capilla, M. \& Del Barrio Gándara, M. (2003). Estructuras de personalidad materna: su implicación en los problemas infantiles. Revista Iberoamericana de Diagnostico y Evaluación Psicológica, 16 (2), 53- 69.

Sendín, M. (2000). Diagnóstico psicológico. Bases conceptuales y guía práctica en los contextos clínico y educativo. Madrid: Psimática.

Shaffer, H. (1990). Making decisions about children. Oxford: Blackwell Publishers.

Wellman, H. (1990). The child's theory of mind. Cambridge, Mass.: MIT Press. 